\title{
ON THE FUNCTIONAL EQUATION $f^{2}=e^{2 \phi_{1}}+e^{2 \phi_{2}}+e^{2 \phi_{3}}$ AND A NEW PICARD THEOREM
}

\author{
BY
}

\section{MARK GREEN}

ABSTRACT. By analogy with E. Borel's reduction of the classical Picard theorem to an analytic statement about linear relations among exponentials of entire functions, a new Picard theorem is proved by considering the functional relation $f^{2}=e^{2 \phi_{1}}+e^{2 \phi_{2}}+e^{2 \phi_{3}}$ for entire functions. The analytic techniques used are those of Nevanlinna theory.

Introduction. An important factor in evoking new interest in value distribution theory has been the re-interpretation of many of its unsolved questions in differential geometric and algebro-geometric language, through the work of Ahlfors, Chern, Griffiths, Kobayashi, Stoll, and Wu. An especially appealing question of this type is whether a holomorphic mapping $f: \mathbf{C} \rightarrow \mathbf{P}_{n}-D$ to complex projective space omitting a hypersurface $D$ with normal crossings and of degree $\geq n+2$ must be algebraically degenerate, in other words whether the image of $f$ must lie in an algebraic hypersurface. For $n=1$, this is just the classical Picard theorem. A few special cases are known for higher $n$, notably if $D$ is a collection of hyperplanes (Borel [2], Cartan [3], Ahlfors [1]; see Green [4] for the case of hyperplanes not in general position) or if $D$ is a Fermat hypersurface $\left\{Z_{0}^{d}+Z_{1}^{d}+\cdots\right.$ $\left.+Z_{n}^{d}=0\right\}$ of high degree (N. Toda [8], Green [5]). The methods employed all have a common feature - the hypothesis is rephrased as a functional equation which is then differentiated several times, and the resulting system of equations is solved. This yields expressions for the coordinate functions of the map in terms of their derivatives, from which a contradiction is obtained by using Nevanlinna theory to show these expressions for the coordinate functions grow too slowly. This contradiction implies that the system of equations is redundant, which is equivalent to the vanishing of a certain Wronskian, forcing a linear relation among the coordinate functions. A complete description of this method may be found in [5].

Unfortunately, the method just sketched does not seem to apply to more general $D$. As a step toward the solution of this apparently difficult problem, we will

Received by the editors September 13, 1973.

AMS (MOS) subject classifications (1970). Primary 30 A70, 32H25; Secondary $32 \mathrm{H} 20$.

Key words and phrases. Picard theorem, value distribution theory, exponential function, Nevanlinna theory, quadric, entire function. 
obtain some results in the case of holomorphic maps $g: \mathbf{C} \rightarrow \mathbf{P}_{2}$ which omit two lines and a conic in a particular relative position. This turns out to involve the study of the functional equation $f^{2}=e^{2 \phi_{1}}+e^{2 \phi_{2}}+e^{2 \phi_{3}}$, where $f$ and $\phi_{i}$ are entire functions.

Analytic preliminaries. A very powerful analytic tool in value distribution theory is the Nevanlinna characteristic function, which we will use in the form introduced by Ahlfors. This technique for expressing the rate of growth of a meromorphic function is beautifully described in Nevanlinna's books [6], [7]; a quick summary of its properties is given in Chapter 1 of [5].

If $f: \mathbf{C} \rightarrow \mathbf{P}_{n}$ is a holomorphic map with homogeneous coordinates $\left(f_{0}, \cdots\right.$ $\left.f_{n}\right)$, we will denote the characteristic function of $f$ by either $T(f, r)$ or $T\left(f_{0}, \cdots f_{n} ; r\right)$. If $f_{0^{0}}, \cdots, f_{n}$ have no common zeros and we set

$$
\mu(Z)=\max _{i} \log \left|f_{i}(Z)\right|,
$$

then

$$
T(f, r)=\frac{1}{2 \pi} \int_{0}^{2 \pi} \mu\left(r e^{i \theta}\right) d \theta-\mu(0) .
$$

If $a=\left(A_{0}, \cdots, A_{n}\right)$ is a hyperplane in $\mathbf{P}_{n}$, and $a \cdot f$ is the function $\Sigma_{i=0}^{n} A_{i} f_{i}$, let $n(f, a, r)$ be the number of zeros of $a \cdot f$ in the disc of radius $r$, counting multiplicities. Then the integrated counting function is defined by

$$
N(f, a, r)=\int_{0}^{r} n(f, a, t) \frac{d t}{t}+n(f, a, 0) \log r .
$$

Nevanlinna's first main theorem states that $N(f, a, r) \leq T(f, r)+O(1)$.

If $g: C \rightarrow C$ is an entire function, when there is no risk of confusion we will denote $T(g, 1 ; r)$ by just $T(g, r)$ and also use $N(g, 0, r)$ to denote the obvious thing.

Many of the functions whose study sparked the classical development of value distribution theory, such as the Weierstrass 8 -function and the Riemann zeta function belong to a subclass of entire functions, those of finite order. A bolomorpbic function of finite order is defined to be one whose Nevanlinna characteristic function is bounded from above by a polynomial in $r$. A consequence of the Hadamard canonical product theorem is that a map of the form $\left(e^{\phi_{1}}, \ldots, e^{\phi_{n}}\right)$, where the $\phi_{i}$ are entire functions, is of finite order if and only if the map can be written $\left(e^{\psi_{1}}, \ldots, e^{\psi_{n}}\right)$ where the $\psi_{i}$ are polynomials.

For convenience, the symbol \| written to the left of an inequality will denote that it holds for all positive values of $r$ except possibly for a set of values having finite total measure. 
We frequently will call upon the Borel lemma (see Borel [2]).

Theorem. Let $e^{\phi_{1}}+e^{\phi_{2}}+\cdots+e^{\phi_{n}}=0$ where the $\phi_{i}$ are entire functions. Tben some $\phi_{i}-\phi_{j}$ is constant, $i \neq j$.

A very strong inequality of Nevanlinna is Nevanlinna's logaritbmic derivative inequality: Let $f$ be a meromorphic function. Then

$$
\| \int_{0}^{2 \pi} \log ^{+}\left|\frac{f^{\prime}(Z)}{f(Z)}\right| d \theta \leq O(\log T(f, r)+\log r)
$$

where $\log ^{+} x$ denotes $\max (\log x, 0)$.

We will use the Abfors defect relations in the case of a curve in $\mathbf{P}_{n-1}$ of the form $\left(e^{\phi_{1}}, \cdots, e^{\phi_{n}}\right)$ where the $\phi_{i}$ are entire functions. It states $\| T\left(e^{\phi} 1, \ldots, e^{\phi} ; r\right) \leq N\left(e^{\phi} 1+e^{\phi_{2}}+\cdots+e^{\phi_{n}}, 0, r\right)+O\left(\log T\left(e^{\phi} 1, \ldots, e^{\phi} ; r\right)\right)$.

Wronskians will play a major role in this paper. The Wronskian of $n$ functions of one variable $A_{1}, \cdots, A_{n}$ is the determinant

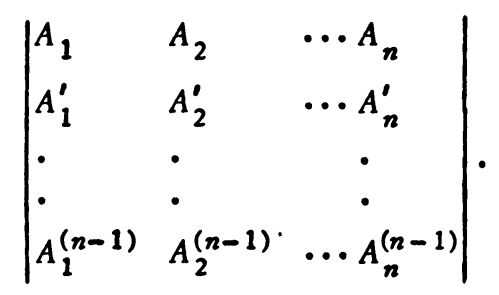

We will denote this by $\left|A_{1} \cdots A_{n}\right|$. It vanishes identically if and only if the functions $A_{1}, \cdots, A_{n}$ are linearly dependent. Two useful identities are

$$
\left|B A_{1} \cdots B A_{n}\right|=B^{n}\left|A_{1} \cdots A_{n}\right|
$$

and

$$
A_{1}^{n-2}\left|A_{1} \cdots A_{n}\right|=|| A_{1} A_{2}|| A_{1} A_{3}|\cdot \cdot| A_{1} A_{n}|| \text {. }
$$

If $A_{1}, \cdots, A_{n}$ are entire functions, it follows from Jensen's theorem and Nevanlinna's logarithmic derivative inequality that

$$
\begin{aligned}
\| N\left(\left|A_{1} A_{2} \cdots A_{n}\right|, 0, r\right) \\
\quad \leq N\left(A_{1}, 0, r\right)+\cdots+N\left(A_{n}, 0, r\right)+O\left(\log T\left(A_{1}, \cdots, A_{n} ; r\right)\right) .
\end{aligned}
$$

Functional relations of the form $f^{d}=e^{d \phi_{1}}+\cdots+e^{d \phi_{n}}$. Let $f_{1} \phi_{1}, \cdots$, $\phi_{n}$ be entire functions on C. A theorem which follows by classical methods is 
Theorem. If $f^{d}=e^{d \phi_{1}}+e^{d \phi_{2}}+\quad+e^{d \phi_{n}}$ and $d \geq n$, then $f=C e^{\phi_{i}}$ for some $i, C$ a constant.

We cannot expect so strong a result for $d<n$. For example,

$$
\left(e^{\phi}+e^{\phi}\right)^{2}=e^{2 \phi_{1}}+e^{\phi_{1}+\phi_{2}+\log 2}+e^{2 \phi_{2}} \text {. }
$$

Hopefully, however, when $d<n$ we should still get that $f$ is a linear combination of exponentials of entire functions. The first nontrivial case is $d=2, n=3$.

When $f^{2}=e^{2 \phi_{1}}+e^{2 \phi_{2}}+e^{2 \phi_{3}}$, it is reasonable to hope that $f$ should be a linear combination of $e^{\phi_{1}}, e^{\phi_{2}}$, and $e^{\phi_{3}}$. In this paper, we will reduce this question to one involving the comparative rates of growth of the Nevanlinna characteristic function $T\left(e^{\phi_{1}}, e^{\phi_{2}}, e^{\phi_{3}} ; r\right)$ and $T\left(e^{-\phi_{1}}, e^{-\phi_{2}}, e^{-\phi_{3}} ; r\right)$. This latter question presents analytic difficulties. Historically, in value distribution theory, results involving delicate analytic questions have first been established for the case of functions of finite order. The theorem will be proved for this case. Specifically, we have:

Theorem. Let $f, \phi_{1}, \phi_{2}, \phi_{3}$ be entire functions on $\mathrm{C}$ satisfying $f^{2}=$ $e^{2 \phi_{1}}+e^{2 \phi_{2}}+e^{2 \phi_{3}}$. Then either

(1) $f$ is a linear combination of $e^{\phi_{1}}, e^{\phi_{2}}$, and $e^{\phi_{3}}$, or

(2) $\|(3 / 2) T_{1}\left(e^{\phi_{1}}, e^{\phi_{2}}, e^{\phi_{3}} ; r\right) \leq T\left(e^{-\phi_{1}}, e^{-\phi_{2}}, e^{-\phi_{3}} ; r\right)+$ $O\left(\log T\left(e^{\phi_{1}}, e^{\phi_{2}}, e^{\phi_{3}} ; r\right)\right)$.

Corollary. If the map $\left(e^{\phi_{1}}, e^{\phi_{2}}, e^{\phi_{3}}\right)$ is of finite order, or equivalently $N(f, 0, r) \leq O\left(r^{n}\right)$ for some $n$, then $f$ is a linear combination of $e^{\phi_{1}}, e^{\phi_{2}}, e^{\phi_{3}}$ whenever $f^{2}=e^{2 \phi_{1}}+e^{2 \phi_{2}}+e^{2 \phi_{3}}$.

We first will prove the theorem and then the corollary.

If $f^{2}=e^{2 \phi_{1}}+e^{2 \phi_{2}}+e^{2 \phi_{3}}$, we obtain a bound on the number of zeros of $\left|f e^{\phi_{1}} e^{\phi_{2}} e^{\phi_{3}}\right|$ unless this function vanishes identically. We have

$$
\begin{aligned}
& f \mid f e^{\phi} 1=e^{\phi_{1}}\left\{\left(\phi_{1}^{\prime}-\phi_{2}^{\prime}\right) e^{2 \phi_{2}}+\left(\phi_{1}^{\prime}-\phi_{3}^{\prime}\right) e^{2 \phi_{3}}\right\}, \\
& f\left|f e^{\phi_{2}}\right|=e^{\phi_{2}}\left\{\left(\phi_{2}^{\prime}-\phi_{1}^{\prime}\right) e^{2 \phi_{1}}+\left(\phi_{2}^{\prime}-\phi_{3}^{\prime}\right) e^{2 \phi_{3}}\right\} ; \\
& f\left|f e^{\phi_{3}}\right|=e^{\phi_{3}}\left\{\left(\phi_{3}^{\prime}-\phi_{1}^{\prime}\right) e^{2 \phi_{1}}+\left(\phi_{3}^{\prime}-\phi_{2}^{\prime}\right) e^{2 \phi_{2}}\right\} .
\end{aligned}
$$

Using, for entire functions $A_{1}, \cdots, A_{n}$, the inequality

$$
\| N\left(\left|A_{1} \cdots A_{n}\right|, 0, r\right) \leq N\left(A_{1}, 0, r\right)+\cdots+N\left(A_{n}, 0, r\right)+O\left(\log T\left(A_{1}, A_{n} ; r\right)\right)
$$

we get 
$\| N\left(|f| f e^{\phi}|, f| f e^{\phi_{2}}|, f| f e^{\phi_{3}}|| 1 ; 0, r\right) \leq N\left(e^{\phi_{1}}\left[\left(\phi_{1}^{\prime}-\phi_{2}^{\prime}\right) e^{2 \phi_{2}}+\left(\phi_{1}^{\prime}-\phi_{3}^{\prime}\right) e^{2 \phi_{3}}\right], 0, r\right)$

$$
\begin{aligned}
+\cdots+ & N\left(e^{\phi_{3}}\left[\left(\phi_{3}^{\prime}-\phi_{1}^{\prime}\right) e^{2 \phi_{1}}+\left(\phi_{3}^{\prime}-\phi_{2}^{\prime}\right) e^{2 \phi_{2}}\right], 0, r\right) \\
& +O\left(\log T\left(e^{\phi_{1}}, e^{\phi_{2}}, e^{\phi_{3}} ; r\right)\right) .
\end{aligned}
$$

Using the Wronskian identities $\left|B A_{1} \cdots B A_{n}\right|=B^{n} \mid A_{1} \cdots A_{n}$ and $\| A_{1} A_{2}|\cdots| A_{1} A_{n}||=A_{1}^{n-2}\left|A_{1} \cdots A_{n}\right|$ to simplify the left-hand side and Nevanlinna's first main theorem and logarithmic inequality to estimate the right-hand side, we obtain

$$
\begin{aligned}
& \| N\left(f^{5}\left|f e^{\phi} e^{\phi}{ }^{\phi} e^{\phi} 3\right|, 0, r\right) \leq T\left(e^{2 \phi}, e^{2 \phi_{3}} ; r\right)+T\left(e^{2 \phi} 1, e^{2 \phi} ; r\right) \\
& +T\left(e^{2 \phi} 1, e^{2 \phi} ; r\right)+O\left(\log T\left(e^{\phi} 1, e^{\phi}, e^{\phi} ; r\right)\right) .
\end{aligned}
$$

This is the crucial estimate.

We remark in general that by the definition of $T$ and $N$ we have $T\left(f_{0}^{d}, \cdots, f_{n}^{d} ; r\right)=d T\left(f_{0}, \cdots, f_{n} ; r\right)$ and $N(f g, 0, r)=N(f, 0, r)+N(g, 0, r)$.

By the Ahlfors defect relations we know

$$
\begin{aligned}
T\left(e^{2 \phi} 1, e^{2 \phi_{2}}, e^{2 \phi} 3 ; r\right) \\
\quad \leq N\left(e^{2 \phi_{1}}+e^{2 \phi_{2}}+e^{2 \phi_{3}}, 0, r\right)+O\left(\log T\left(e^{\phi_{1}}, e^{\phi_{2}}, e^{\phi_{3}} ; r\right)\right)
\end{aligned}
$$

from which follows

$$
T\left(e^{\phi_{1}}, e^{\phi_{2}}, e^{\phi_{3}} ; r\right) \leq N(f, 0, r)+O\left(\log T\left(e^{\phi_{1}}, e^{\phi_{2}}, e^{\phi_{3}} ; r\right)\right) .
$$

$A$ weaker version of the main inequality says

$$
\begin{aligned}
\| S N(f, 0, r) \leq & 2\left[T\left(e^{\phi_{2}}, e^{\phi_{3}}, r\right)+T\left(e^{\phi_{1}}, e^{\phi_{3}} ; r\right)+T\left(e^{\phi_{1}}, e^{\phi_{2}} ; r\right)\right] \\
& +O\left(\log T\left(e^{\phi_{1}}, e^{\phi_{2}}, e^{\phi_{3}} ; r\right)\right)
\end{aligned}
$$

provided $\left|f e^{\phi_{1}} e^{\phi_{2}} e^{\phi_{3}}\right|$ does not vanish identically.

So

$$
\begin{aligned}
\| S T\left(e^{\phi_{1}}, e^{\phi_{2}}, e^{\phi_{3}} ; r\right) \leq & 2\left[T\left(e^{\phi_{2}}, e^{\phi_{3}} ; r\right)+T\left(e^{\phi_{1}}, e^{\phi_{3}} ; r\right)+T\left(e^{\phi_{1}}, e^{\phi_{2}} ; r\right)\right] \\
& +O\left(\log T\left(e^{\phi_{1}}, e^{\phi_{2}}, e^{\phi_{3}} ; r\right)\right) .
\end{aligned}
$$

Using the definition of $T$, we see

$$
\begin{aligned}
& T\left(e^{\phi_{1}}, e^{\phi_{2}} ; r\right)+T\left(e^{\phi_{1}}, e^{\phi_{3}} ; r\right)+T\left(e^{\phi_{2}}, e^{\phi_{3}} ; r\right) \\
& \quad=T\left(e^{2 \phi_{1}+\phi_{2}}, e^{2 \phi_{1}+\phi_{3}}, e^{2 \phi_{2}+\phi_{1}}, e^{2 \phi_{2}+\phi_{3}}, e^{2 \phi_{3}+\phi_{1}}, e^{2 \phi_{3}+\phi_{2}}, e^{\phi_{1}+\phi_{2}+\phi_{3}} ; r\right) \\
& \quad=T\left(e^{\phi_{1}}, e^{\phi_{2}}, e^{\phi_{3}} ; r\right)+T\left(e^{\phi_{1}+\phi_{2}}, e^{\phi_{1}+\phi_{3}}, e^{\phi_{2}+\phi_{3}} ; r\right) .
\end{aligned}
$$


So we have

$$
\| 3 T\left(e^{\phi_{1}}, e^{\phi_{2}}, e^{\phi_{3}} ; r\right)
$$

$$
\leq 2 T\left(e^{\phi_{1}+\phi_{2}}, e^{\phi_{1}+\phi_{3}}, e^{\phi_{2}+\phi_{3}} ; r\right)+O\left(\log T\left(e^{\phi_{1}}, e^{\phi_{2}}, e^{\phi_{3}} ; r\right)\right) .
$$

Since $T(A, B, C ; r)=T\left(e^{\phi} A, e^{\phi} B, e^{\phi} C ; r\right)$ by definition, we have

$$
T\left(e^{\phi_{2}+\phi_{3}}, e^{\phi_{1}+\phi_{3}}, e^{\phi_{1}+\phi_{2}} ; r\right)=T\left(e^{-\phi_{1}}, e^{-\phi_{2}}, e^{-\phi_{3}} ; r\right) \text {. }
$$

So we conclude that either $\left|f e^{\phi_{1}} e^{\phi_{2}} e^{\phi_{3}}\right|$ vanishes identically, in which case $f, e^{\phi_{1}}, e^{\phi_{2}}, e^{\phi_{3}}$ are linearly dependent, or else the inequality (2) of the conclusion of the theorem holds. If the linear dependence above does not involve f. then by the Borel lemma some $e^{\phi_{i}} / e^{\phi_{j}}$ is constant, $i \neq j$, and we can consolidate our functional relation to one of the form $f^{2}=e^{2 \phi_{1}}+e^{2 \phi_{2}}$ and the desired conclusion follows by a result mentioned at the beginning of this section. This completes the proof of the theorem.

It remains to prove the corollary. We do this by showing that for finite order functions we have

$$
T\left(e^{-\phi_{1}}, e^{-\phi_{2}}, e^{-\phi_{3}} ; r\right) \leq T\left(e^{\phi_{1}}, e^{\phi_{2}}, e^{\phi_{3}} ; r\right)+O\left(T\left(e^{\phi_{1}}, e^{\phi_{2}}, e^{\phi_{3}} ; r\right)\right) .
$$

In the finite order case, we may choose $\phi_{1}, \phi_{2}, \phi_{3}$ to be polynomials in $Z$. If $d$ is the largest degree to occur, let $\phi_{i}=\lambda_{i} Z^{d}+p_{i}(Z)$, deg $p_{i} \leq d-1$, where not all $\lambda_{i}$ are zero nor are all $\lambda_{i}$ equal.

Then by the definition of $T$ we see

$$
\left|T\left(e^{\phi_{1}}, e^{\phi_{2}}, e^{\phi_{3}} ; r\right)-T\left(e^{\lambda_{1} z^{d}}, e^{\lambda_{2} z^{d}}, e^{\lambda_{3} z^{d}} ; r\right)\right| \leq o\left(T\left(e^{\phi_{1}}, e^{\phi_{2}}, e^{\phi_{3}} ; r\right)\right) \text {. }
$$

Likewise,

Therefore it will suffice to show

$$
\begin{gathered}
\left|T\left(e^{-\phi_{1}}, e^{-\phi_{2}}, e^{-\phi_{3}} ; r\right)-T\left(e^{-\lambda_{1} z^{d}}, e^{-\lambda_{2} z^{d}}, e^{-\lambda_{3} z^{d}} ; r\right)\right| \\
<o\left(T\left(e^{\phi_{1}}, e^{\phi_{2}}, e^{\phi_{3}}, r\right)\right) .
\end{gathered}
$$

$$
T\left(e^{-\lambda_{1} z^{d}}, e^{-\lambda_{2} z^{d}}, e^{-\lambda_{3} z^{d}} ; r\right) \leq T\left(e^{\lambda_{1} z^{d}}, e^{\lambda_{2} z^{d}} ; e^{\lambda_{3} z^{d}} ; r\right) \text {. }
$$

By the change of variable $w=Z^{d}$ and the definition of $T$ we can reduce to the case $d=1$. A direct calculation done in Ahlfors [1] gives

$$
T\left(e^{\lambda_{1} Z}, e^{\lambda_{2} Z}, e^{\lambda_{3} Z} ; r\right)=(2 \pi r)^{-1}\left(\left|\lambda_{1}-\lambda_{2}\right|+\left|\lambda_{1}-\lambda_{3}\right|+\left|\lambda_{2}-\lambda_{3}\right|\right)+O(1)
$$

and thus

$$
T\left(e^{-\lambda_{1} z}, e^{-\lambda_{2} z}, e^{-\lambda_{3} z} ; r\right)=(2 \pi)^{-1}\left(\left|\lambda_{1}-\lambda_{2}\right|+\left|\lambda_{1}-\lambda_{3}\right|+\left|\lambda_{2}-\lambda_{3}\right|\right)+O(1) \text {, }
$$

which completes the proof. 
Algebro-geometric interpretation and open problems. Very little is presently known about when the image of a homomorphic curve $f: C \rightarrow \mathrm{P}_{n}-D$ omitting a hypersurface $D$ with normal crossings and degree $\geq n+2$ must lie in a proper algebraic hypersurface of $\mathbf{P}_{n}$, unless $D$ is a union of hyperplanes or is a Fermat variety. One can parlay these theorems into somewhat more general ones, for example if $D$ is the union of $n+2$ hypersurfaces of arbitrary degree, without any new analytic results. Even so, it is not at all clear that there should be a theorem for a general $D$.

The purpose of this paper is to show that further results can be obtained through new analytic techniques. The main theorem on the relation $f^{2}=e^{2 \phi_{1}}+$ $e^{2 \phi_{2}}+e^{2 \phi_{3}}$ may be interpreted in the following geometric way:

Theorem. Let $g: \mathbf{C} \rightarrow \mathbf{P}_{2}$ be a bolomorpbic curve of finite order omitting the two lines $\left\{Z_{1}=0\right\}$ and $\left\{Z_{2}=0\right\}$ and the conic $\left\{Z_{0}^{2}+Z_{1}^{2}+Z_{2}^{2}=0\right\}$. Then the image of $g$ lies in a line or a conic.

Remark. The curve $\left(1-e^{Z}, \sqrt{2} e^{Z / 2}, i e^{Z}\right)$ shows that we cannot eliminate the case of a conic from the conclusion (the image cannot lie in a line by the Borel lemma).

Proof. If $g=\left(g_{0}, g_{1}, g_{2}\right)$ where the $g_{i}$ never all vanish simultaneously, then as a never-vanishing entire function is the exponential of some entire function, there must exist $\phi_{1}, \phi_{2}, \phi_{3}$ so

$$
\sqrt{-1} g_{1}=e^{\phi}, \quad \sqrt{-1} g_{2}=e^{\phi}, \quad g_{0}^{2}+g_{1}^{2}+g_{2}^{2}=e^{2 \phi_{3}}
$$

so $g_{0}^{2}=e^{2 \phi_{1}}+e^{2 \phi_{2}}+e^{2 \phi_{3}}$.

If $g$ is of finite order, it is easy to see $\left(e^{\phi_{1}}, e^{\phi_{i}}, e^{\phi_{3}}\right)$ is also. So we may conclude $g_{0}$ is a linear combination of $e^{\phi_{1}}, e^{\phi_{2}}$, and $e^{\phi_{3}}$, say $g_{0}=$ $a_{1} e^{\phi_{1}}+a_{2} e^{\phi_{2}}+a_{3} e^{\phi_{3}}$. If $a_{3}=0$, we have that the image of $g$ lies in a line. Otherwise

$$
\left(\left(g_{0}-a_{1} \sqrt{-1} g_{1}-a_{2} \sqrt{-1} g_{2}\right) / a_{3}\right)^{2}=g_{0}^{2}+g_{1}^{2}+g_{2}^{2}
$$

which shows the image of $g$ lies in a conic.

The more general situation of a holomorphic curve in $\mathbf{P}_{2}$ omitting any two lines and a conic in general position reduces to a relation of the form $f^{2}=$ $e^{2 \phi_{1}}+e^{2 \phi_{2}}+e^{2 \phi_{3}}+e^{2 \phi_{4}}$. Such a relation cannot be dealt with by the same proof as given for a relation of length three, because it is in fact not necessary that $f$ be a linear combination of $e^{\phi_{1}}, e^{\phi_{2}}, e^{\phi_{3}}$, and $e^{\phi_{4}}$. This relation can be treated by an extension of the method of this paper. In many cases, these techniques give interesting but incomplete information. 
It is easy to show in general that

$$
1 / 2 T\left(e^{-\phi_{1}}, e^{-\phi_{2}}, e^{-\phi_{3}} ; r\right) \leq T\left(e^{\phi_{1}}, e^{\phi_{2}}, e^{\phi_{3}} ; r\right) \leq 2 T\left(e^{-\phi_{1}}, e^{-\phi_{2}}, e^{-\phi_{3}} ; r\right)
$$

for arbitrary entire functions $\phi_{1}, \phi_{2}, \phi_{3}$. Insofar as I know, we should have

$$
\|\left|T\left(e^{\phi_{1}}, e^{\phi_{2}}, e^{\phi_{3}} ; r\right)-T\left(e^{-\phi_{1}}, e^{-\phi_{2}}, e^{-\phi_{3}} ; r\right)\right| \leq O\left(T\left(e^{\phi_{1}}, e^{\phi_{2}}, e^{\phi_{3}} ; r\right)\right)
$$

in all cases, and not just in the finite order case. This analytic fact would imply the main theorem with the hypothesis of finite order dropped.

By an argument more intricate than that in this paper, I believe it can be shown that if $f^{d}=e^{d \phi_{1}}+\cdots+e^{d \phi_{n}}$, then either $f$ is a linear combination of exponentials of entire functions or

$$
\begin{gathered}
\|\left|T\left(e^{\phi_{1}+\phi_{2}}, e^{\phi_{1}+\phi_{3}}, \ldots, e^{\phi_{n-1}+\phi_{n}} ; r\right)-2 T\left(e^{\phi_{1}}, \ldots, e^{\phi} n\right)\right| \\
\leq O\left(\log T\left(e^{\phi_{1}}, \ldots, e^{\phi_{n}} ; r\right)\right) .
\end{gathered}
$$

Acknowledgements. I am grateful for the help and encouragement of James Carlson, S. S. Chern, Phillip Griffiths, and H. Wu.

\section{BIBLIOGRAPHY}

1. .L. Ahlfors, The theory of meromorphic curves, Acta Soc. Sci. Fenn. Nova Ser. A 3 (1941), no. 4, 31 pp. MR 2, 357.

2. E. Borel, Sur les zéros des fonctions entières, Acta Math. 20 (1887), 357-396.

3. H. Cartan, Sur les zéros des combinaisons linéaires de p fonctions holomorphes données, Mathematica (Cluj) 7 (1933).

4. M. Green, Holomorphic maps into complex projective space omitting hyperplanes, Trans. Amer. Math. Soc. 169 (1972), 89-103.

5. - Some Picard theorems for holomorphic maps to algebraic varieties, Amer. J. Math. (to appear).

6. R. Nevanlinna, Eindeutige analytische Funktionen, Die Grundlehren der math. Wissenschaften, Band 46, Springer-Verlag, Berlin, 1936; English transl., Die Grundlehren der math. Wissenschaften, Band 162, Springer-Verlag, Berlin and New York, 1970. MR 43 \#5003.

7. Le théorème de Picard-Borel et la théorie des fonctions méromorphes, Gauthier-Villars, Paris, 1929.

8. N. Toda, On the functional equation $\Sigma_{i=0}^{p} a_{i} f_{i}^{n_{i}}=1$, Tôhuku Math. J. (2) 23 (1971), 289-299. MR 45 \#551. 94720

DEPARTMENT OF MATHEMATICS, UNIVERSITY OF CALIFORNIA, BERKELEY, CALIFORNIA 